\title{
Detection of superior genotype of fatty acid synthase in Korean native cattle by an environment-adjusted statistical model
}

\author{
Jea-Young Lee ${ }^{1, a}$, Dong-Yep $\mathrm{Oh}^{2, a}$, Hyun-Ji Kim ${ }^{1}$, Gab-Sue Jang ${ }^{3}$, and Seung-Uk Lee ${ }^{4, *}$
}

* Corresponding Author: Seung-Uk Lee Tel: +82-10-3811-1830, Fax: +82-54-638-6013,

E-mail: ceels@nus.edu.sg

'Department of Statistics, Yeungnam University, Gyeongsan 712-749, Korea

${ }^{2}$ Livestock Research institute, Yeongju 750-871, Korea

${ }^{3}$ Department of Life Sciences, Yeungnam University,

Gyeongsan 712-749, Korea

${ }^{4}$ Department of Civil and Environmental Engineering, National University of Singapore, Singapore 117576 Singapore

a These authors contributed equally to this work. Submitted Apr 4, 2016; Revised Sept 27, 2016; Accepted Feb 1, 2017
Objective: This study examines the genetic factors influencing the phenotypes (four economic traits:oleic acid [C18:1], monounsaturated fatty acids, carcass weight, and marbling score) of Hanwoo.

Methods: To enhance the accuracy of the genetic analysis, the study proposes a new statistical model that excludes environmental factors. A statistically adjusted, analysis of covariance model of environmental and genetic factors was developed, and estimated environmental effects (covariate effects of age and effects of calving farms) were excluded from the model.

Results: The accuracy was compared before and after adjustment. The accuracy of the best single nucleotide polymorphism (SNP) in C18:1 increased from $60.16 \%$ to $74.26 \%$, and that of the two-factor interaction increased from $58.69 \%$ to $87.19 \%$. Also, superior SNPs and SNP interactions were identified using the multifactor dimensionality reduction method in Table 1 to 4. Finally, high- and low-risk genotypes were compared based on their mean scores for each trait.

Conclusion: The proposed method significantly improved the analysis accuracy and identified superior gene-gene interactions and genotypes for each of the four economic traits of Hanwoo.

Keywords: Adjusted Model; Analysis of Covariance (ANCOVA) Model; Environmental Factor; Fatty Acid Synthase; Genetic Factor

\section{INTRODUCTION}

Beef quality depends on fatty acid composition (FAC) and the degree of marbling. Beef's delicious flavor comes from its carcass fat with small amounts of saturated fatty acids (SFAs) and large amounts of monounsaturated fatty acids (MUFAs) [1-3]. The MUFA:SFA ratio is an indirect factor determining the taste of beef [4]. Oleic acid (C18:1), a major MUFA in beef fat, is considered a vital ingredient in the aroma of cooked beef [1,5]. For high-quality beef, it is important to improve its FAC, particularly its C18:1. According to the livestock-rating system of the Korea Institute for Animal Products Quality Evaluation, carcass weight (CWT) and marbling score (MS) are major indicators of beef quality in carcass traits.

In particular, fatty acid synthase (FASN), which is abundantly expressed in adipose tissue, is a complex homodimeric enzyme that regulates the biosynthesis of long-chain fatty acids [6]. We recently examined the genetic relationships between the FAC of beef and multiple nucleotide sequence variants in the gene encoding FASN [7]. We found that five single-nucleotide polymorphism (SNP) variants, namely g.12870 T>C, g.13126 T>C, g.15532 C>A, g.16907 T>C, and g.17924 G>A in exon regions $23,24,34,37$, and 39 , respectively, were associated with the composition of unsaturated fatty acids and MUFAs in the adipose tissue of Japanese Black cattle and Korean native cattle $(\mathrm{p}<0.05)$. In addition, the g.17250-17251 AT Indel, g.16907 T>C, g.15532 $\mathrm{C}>\mathrm{A}, \mathrm{g} .15603 \mathrm{G}>\mathrm{A}$, and g.17924 G>A variants have been found to be associated with the myristic acid content of adipose tissue in the beef cattle crossbred between Jersey and Limousin 
$(p<0.05)$ [8]. Another crossbred between Japanese Black cattle and Limousin has been found to show the association between FAC and g.16024 A $>\mathrm{G}$ and g.16039 $\mathrm{T}>\mathrm{C}(\mathrm{p}<0.05)$ [9]. In addition, g.17924 G>A and g.18663 T>C have been found to be associated with the oleic acid content of adipose tissue in Japanese Black cattle $(\mathrm{p}<0.05)$ [10]. Based on these findings, the FASN gene, which is closely related to C18:1 as an important factor influencing the beef flavor, may play an important role in improving beef quality.

This study identifies the SNPs in exons of the FASN gene influencing C18:1, MUFAs, CWT, and MS in Hanwoo (Korean native cattle). The FASN gene is significantly related to FAC and carcass traits $[7,11]$. The first important point is that most traits of economic importance in livestock are multifactorial in nature, and are thus influenced by multiple genes and their interactions with environmental factors. Enhancing the accuracy of genetic analysis necessitates a statistical model that excludes environmental effects. Therefore, this study proposes an analysis of covariance (ANCOVA) model that includes environmental and genetic factors influencing the phenotype of Hanwoo and uses a new adjusted model that eliminates the estimated values of environmental factors. In addition, the study employs the multifactor dimensionality reduction (MDR) method to test the main and interaction effects of multiple SNPs on the meat quality of Hanwoo and compares the analysis accuracy between the adjusted and unadjusted models. Finally, the study explores superior genotype groups based on interactions between SNPs in exons of the FASN gene.

\section{MATERIALS AND METHODS}

\section{Phenotypes and SNP genotyping}

A total of 513 Hanwoo cattle were bred at 17 farms in Gyeongsangbuk-do, Korea and fed according to each farmer's feeding program. In general, they were weaned and castrated at 6 months of age, fed with growth stage feed for 18 months, and then fed a highly concentrated diet for their last 6 months. All steers were slaughtered at $941 \pm 72$ days of age with an average CWT of 427.25 $\pm 43.281 \mathrm{~kg}$. The MS was measured $24 \mathrm{~h}$ after slaughter. First, the carcasses were dissected at the last rib and the first lumber vertebra according to the Animal Product Grading System of Korea. MS ranged from 1 to 9 , where a higher score indicates more abundant intramuscular fat. FAC was analyzed using gas-chromatography (PerkinElmer, Inc., Waltham, MA, USA) $[7,12,13]$. The composition of the fatty acids, MS, and CWT were used to establish phenotypes for the genetic association analysis of Hanwoo.

Total genomic DNA was extracted from the longissimus muscle by using the LaboPass TM Tissue Mini kit (Cosmo Genetech, Seoul, Korea). Five polymorphic exonic SNPs of the FASN gene in GenBank (Accession no. AF285607) were genotyped, according to our previous study [7]. We previously examined the genetic relationships between the FAC of beef and multiple nucleotide sequence variants in the FASN gene, and five variants, namely g.12870 T>C, g.13126 T>C, g.15532 C>A, g.16907 T>C, and g.17924 G>A in exon regions $23,24,34,37$, and 39, respectively, were found to be associated with the composition of unsaturated fatty acids [7].

\section{A statistical ANCOVA model of the economic traits of Hanwoo}

The economic traits of Hanwoo are affected by individual SNPs, calving farms, age, etc. In this study, we used C18:1, MUFAs, MS, and CWT as economic traits and SNPs in exons of the FASN gene, calving farms and age as factors. The relationship between economic traits and factors can be expressed as the following ANCOVA model:

$$
\begin{aligned}
Y_{k}= & \mu+\alpha_{0}\left(A_{k}-\bar{A}\right)+\alpha_{1} F_{1 k}+\cdots+\alpha_{l-1} F_{l-1, k} \\
& +\beta_{1} G_{1 k}+\cdots+\beta_{m} G_{m k}+\epsilon_{k} \\
i= & 1,2, \cdots, l ; j=1,2, \cdots, m ; k=1,2, \cdots, n,
\end{aligned}
$$

where $Y_{k}$ indicates the economic traits of Hanwoo $k, \mu$ is the overall mean, $A_{k}$ the covariate for age in days at slaughter, $\bar{A}$ the mean of $A_{k}, \alpha_{0}$ the covariate effect, $F_{i k}$ an indicator variable for calving farm $i$ (17 classes), $\alpha_{i}$ the fixed effect of $F_{i k}, G_{j k}$ the genotype $j$, $\beta_{j}$ the fixed effect of $G_{j k}$, and $\epsilon_{k}$ a random residual assumed to have an independent and identical normal distribution. In a statistical model, if a qualitative (categorical) variable has more than two classes (17 calving farms), we require additional indicator variables in the model [14]. Since the calving farm variable has 17 classes, we require 16 indicator variables.

Define the binary $F_{i k}$ as follows:

$$
F_{i k}=\left\{\begin{array}{ll}
1, & \text { if the farm of the kth Hanwoo is ith, } \\
0, & \text { otherwise. }
\end{array} \quad(i=1,2, \cdots, l-1)\right.
$$

The above model can be briefly expressed as follows:

$$
Y=\mu+E \alpha+G \beta+\epsilon
$$

The economic traits of Hanwoo can be treated as the $Y$ matrix, and environmental and genetic factors, as the $E$ and $G$ matrices, respectively:

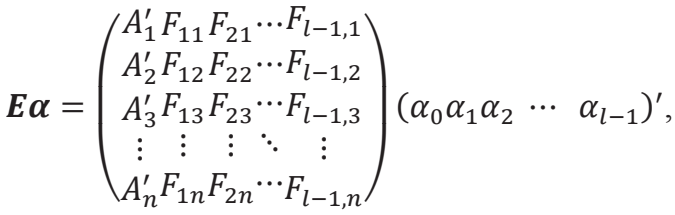

$$
\begin{aligned}
& \boldsymbol{G} \boldsymbol{\beta}=\left(\begin{array}{cccc}
G_{11} & G_{21} & \cdots & G_{m 1} \\
G_{12} & G_{22} & \cdots & G_{m 2} \\
G_{13} & G_{23} & \cdots & G_{m 3} \\
\vdots & \vdots & \ddots & \vdots \\
G_{1 n} G_{2 n} & \cdots & G_{m n}
\end{array}\right)\left(\beta_{1} \beta_{2} \beta_{3} \cdots \beta_{m}\right)^{\prime}
\end{aligned}
$$




$$
A^{\prime}=A_{k}-\bar{A}, i=1,2, \cdots, l ; j=1,2, \cdots, m ; k=1,2, \cdots, n,
$$

where the age in days at slaughter and the calving farm are environmental factors and SNPs are genetic factors.

Here environmental factors were excluded from the ANCOVA model because a statistical model was required only for genetic factors. Statistical analysis was conducted using SPSS v22.0 (SPSS Inc., Chicago, IL, USA):

$$
\boldsymbol{Z}=\boldsymbol{Y}-\boldsymbol{E} \widehat{\boldsymbol{\alpha}},
$$

where $\boldsymbol{Z}, \boldsymbol{Y}, \widehat{\boldsymbol{\alpha}}$ are

$$
\boldsymbol{Z}=\left(\begin{array}{c}
\operatorname{adj}\left(Y_{1}\right) \\
\operatorname{adj}\left(Y_{2}\right) \\
\operatorname{adj}\left(Y_{3}\right) \\
\vdots \\
\operatorname{adj}\left(Y_{n}\right)
\end{array}\right), \boldsymbol{Y}=\left(\begin{array}{c}
Y_{1} \\
Y_{2} \\
Y_{3} \\
\vdots \\
Y_{n}
\end{array}\right), \widehat{\boldsymbol{\alpha}}=\left(\begin{array}{c}
\hat{\alpha}_{0} \\
\hat{\alpha}_{1} \\
\hat{\alpha}_{2} \\
\vdots \\
\hat{\alpha}_{l-1}
\end{array}\right)
$$

The above model can be defined as an "adjusted model" that excludes environmental factors. Superior SNPs and SNP-SNP interactions influencing economic traits were identified by applying the MDR method to adjusted data $(\boldsymbol{Z})$.

\section{Multifactor dimensionality reduction analysis}

The MDR method is nonparametric and model-free and has been implemented in case-control studies [15]. For application to continuous data, each phenotype was divided into the case group $(\mathrm{Y}$ $=1)$ and the control group $(\mathrm{Y}=0)$ by using the $\mathrm{k}$-means clustering method. The MDR method classifies the phenotypes into two groups: high and low. Each individual is assigned to a cell (e.g., if there are two SNPs with three genotypes per SNP, then there are nine possible cells), and each cell is defined as a high- or lowrisk group.

Then the error is

$$
\text { Error }=\frac{{ }^{N}(Y=0 \mid \text { high })^{-N}(Y=1 \mid \text { low })}{N_{\text {total }}} .
$$

The MDR steps were conducted according to the procedure we have previously reported [7]. The procedure is described here briefly as follows:

Step 1. Data were randomly divided into 10 equal parts: one testing set and nine training sets as part of the cross-validation procedure.

Step 2. A set of $n$ SNPs was selected from the pool of all SNPs. Step 3. Based on the observed level of each $n$, steers were partitioned into classes referred to as cells. If $n=2$, then a set of two SNPs was selected, and because one SNP had three genotypes, there were $3^{2}=9$ possible cells. Case-control ratios were cal- culated for each cell.

Step 4. The case-control ratio of total data was used as the threshold such that a cell with a higher case-control ratio than the threshold was labeled as high and the remainder, as low. Step 5. The MDR model with the smallest test data error was chosen among all two-factor combinations.

Step 6. To evaluate the predictive ability of the model, the test data error was estimated using a 10 -fold cross-validation method.

These six steps were repeated for each possible combination of given $n$. The model with the minimum test data error was selected as the best model. However, statistical significance was not determined by the test data error for the selected best model. Therefore, $t$-tests were conducted, and Cohen's $d$ was calculated to determine empirical significance thresholds by applying the same MDR method.

\section{Effect size}

The p-value is commonly used to describe test results. The weakness of the power of a statistical test is that the p-value is influenced by sample size. Therefore, the null hypothesis can be rejected in most cases if the sample is very large, even when the real difference is negligible. To overcome this weakness, effect size can be used in the statistical analysis. Effect size can be defined as the "standardized measure of the size of the mean difference of the relationship among the study groups". In this regard, Cohen's d was used for effect size to indicate standardized differences for a comparison of two independent means [16,17]:

$$
\text { Cohen's } d=\frac{\left(\mu_{\text {high }}-\mu_{\text {low }}\right)}{\sqrt{\frac{\sigma_{\text {high }}^{2}+\sigma_{\text {low }}^{2}}{2}}},
$$

where the bigger the difference between two independent means, the bigger the effect size.

\section{RESULTS}

In this study, the used genetic factors were five individual SNPs (g.12870 T>C, g.13126 T>C, g.15532 C>A, g.16907 T>C, and g. $17924 \mathrm{G}>\mathrm{A}$ ) in the exon of the FASN gene that were associated with FAC of Korean cattle and not deviated from the HardyWeinberg equilibrium [7].

Tables 1 to 4 summarize the accuracy of gene-gene interaction models for the four economic traits. This accuracy was determined before and after environmental factors were adjusted for. According to the results, the higher-order interaction model was significantly more accurate than the SNP model, and adjusted data were more accurate than raw data.

First, the accuracy was compared before and after adjustment. The accuracy of the best SNP in C18:1 increased from 60.16\% 
Table 1. The best SNP in five-factor interactions of the C18:1 base in the MDR method for Korean native cattle

\begin{tabular}{|c|c|c|c|c|}
\hline Adjustment & Model & Training acc. ${ }^{1)}$ & Test acc. ${ }^{2)}$ & $\mathrm{CVC}^{3)}$ \\
\hline \multirow[t]{5}{*}{ Before } & g. $13126 \mathrm{~T}>\mathrm{C}$ & 0.6016 & 0.6016 & $10 / 10$ \\
\hline & g. $13126 \mathrm{~T}>\mathrm{C}, \mathrm{g} .15532 \mathrm{C}>\mathrm{A}$ & 0.6235 & 0.5869 & $9 / 10$ \\
\hline & g.13126 T>C, g.15532 C>A, g.16907 T >C & 0.6407 & 0.6176 & $10 / 10$ \\
\hline & g.12870 T>C, g.13126 T>C, g.15532 C>A, g.16907 T>C & 0.6517 & 0.6300 & $10 / 10$ \\
\hline & g.12870 T>C, g.13126 T>C, g.15532 C>A, g.16907 T>C, g.17921 G>A & 0.6565 & 0.6317 & $10 / 10$ \\
\hline \multirow[t]{5}{*}{ After } & g. $13126 \mathrm{~T}>\mathrm{C}$ & 0.7426 & 0.7426 & $10 / 10$ \\
\hline & g. $13126 \mathrm{~T}>\mathrm{C}, \mathrm{g} .15532 \mathrm{C}>\mathrm{A}$ & 0.8719 & 0.8719 & $10 / 10$ \\
\hline & g.13126 T>C, g. 15532 C >A, g.17924 G >A & 0.9886 & 0.9829 & $8 / 10$ \\
\hline & g.12870 T>C, g.13126 T>C, g.15532 C>A, g.17924 G>A & 1.0000 & 0.9956 & $10 / 10$ \\
\hline & g.12870 T>C, g.13126 T>C, g.15532 C>A, g.16907 T>C, g.17924 G>A & 1.0000 & 0.9846 & $10 / 10$ \\
\hline
\end{tabular}

SNP, single nucleotide polymorphism; MDR, multifactor dimensionality reduction.

${ }^{1)}$ Training-balanced accuracy. ${ }^{2)}$ Test-balanced accuracy. ${ }^{3)}$ Cross-validation consistency.

to $74.26 \%$, and that of the two-factor interaction increased from $58.69 \%$ to $87.19 \%$. In addition, the accuracy of MUFAs, MS, and CWT increased by $16.78 \%$ p, $28.98 \%$ p, and $39.98 \%$ p, respectively, in the best SNP and by $27.73 \%$ p, $43.53 \%$ p, and $45.75 \%$ p in the two-factor interaction. Therefore, adjusted data were used to identify gene-gene interaction effects.

Second, superior SNPs and SNP interactions were identified using the MDR method. In the case of C18:1 (Table 1) and MUFAs (Table 2), g.13126 T>C had the highest score for both training- and test-balanced accuracy (74.26\% for C18:1 and 74.61\% for MUFAs). In terms of the two-factor interaction, g.13126 T>C and g.15532 C>A had the highest training-and test-balanced accuracy (87.19\% for C18:1 and 88.10\% for MUFA), with a crossvalidation consistency of 10 out of 10 . For MS, the SNP g.12870 $\mathrm{T}>\mathrm{C}$ showed the highest accuracy at $80.75 \%$, and the two-factor interaction model g.12870 $\mathrm{T}>\mathrm{C}$ and g.15532 $\mathrm{C}>\mathrm{A}$ showed the highest testing-balanced accuracy at $97.12 \%$ (Table 3 ). The best SNP for CWT was g.12870 T>C at 84.4\% accuracy, and the two-

Table 2. The best SNP in five-factor interactions of the MUFA base in the MDR method for Korean native cattle

\begin{tabular}{|c|c|c|c|c|}
\hline Adjustment & Model & Training acc. ${ }^{1)}$ & Test acc. ${ }^{2)}$ & $\mathrm{CVC}^{3)}$ \\
\hline \multirow[t]{5}{*}{ Before } & g.13126 T>C & 0.5871 & 0.5783 & $10 / 10$ \\
\hline & g.13126 T>C, g.15532 C >A & 0.6127 & 0.6037 & $10 / 10$ \\
\hline & g.13126 T>C, g.15532 C >A, g. $16907 \mathrm{~T}>\mathrm{C}$ & 0.6221 & 0.5633 & $6 / 10$ \\
\hline & g.12870 T>C, g.13126 T>C, g.15532 C >A, g. $16907 \mathrm{~T}>\mathrm{C}$ & 0.6296 & 0.5715 & $8 / 10$ \\
\hline & g.12870 T>C, g.13126 T>C, g.15532 C>A, g.16907 T>C, g.17921 G>A & 0.6337 & 0.5660 & $10 / 10$ \\
\hline \multirow[t]{5}{*}{ After } & g.13126 T>C & 0.7461 & 0.7461 & $10 / 10$ \\
\hline & g.13126 T>C, g.15532 C >A & 0.8810 & 0.8810 & $10 / 10$ \\
\hline & g.13126 T>C, g.15532 C>A, g.17924 G>A & 0.9957 & 0.9935 & $10 / 10$ \\
\hline & g.12870 T>C, g.13126 T>C, g.15532 C >A, g.17924 G >A & 1.0000 & 0.9957 & $10 / 10$ \\
\hline & g.12870 T>C, g.13126 T>C, g.15532 C >A, g.16907 T>C, g.17924 G>A & 1.0000 & 0.9848 & $10 / 10$ \\
\hline
\end{tabular}

SNP, single nucleotide polymorphism; MUFA, monounsaturated fatty acid; MDR, multifactor dimensionality reduction.

${ }^{1)}$ Training-balanced accuracy. ${ }^{2)}$ Test-balanced accuracy. ${ }^{3)}$ Cross-validation consistency.

Table 3. The best SNP in five-factor interactions of the MS base in the MDR method for Korean native cattle

\begin{tabular}{|c|c|c|c|c|}
\hline Adjustment & Model & Training acc. $^{1)}$ & Test acc. $^{2)}$ & $\mathrm{CVC}^{3)}$ \\
\hline \multirow[t]{5}{*}{ Before } & g.15532 C>A & 0.5459 & 0.5177 & $10 / 10$ \\
\hline & g.12870 T>C, g.15532 C >A & 0.5689 & 0.5359 & $8 / 10$ \\
\hline & g. 12870 T > C, g. 15532 C >A, g. 16907 T > C & 0.5836 & 0.5092 & $7 / 10$ \\
\hline & g.12870 T>C, g.13126 T>C, g.15532 C > A, g.16907 T>C & 0.5957 & 0.5205 & $10 / 10$ \\
\hline & g.12870 T>C, g.13126 T>C, g.15532 C>A, g.16907 T>C, g.17924 G>A & 0.6019 & 0.5278 & $10 / 10$ \\
\hline \multirow[t]{5}{*}{ After } & g.12870 T>C & 0.8075 & 0.8075 & $10 / 10$ \\
\hline & g.12870 T>C, g.15532 C >A & 0.9712 & 0.9712 & $10 / 10$ \\
\hline & g.12870 T>C, g.13126 T>C, g.15532 C>A & 0.9915 & 0.9858 & $10 / 10$ \\
\hline & g.12870 T>C, g.13126 T>C, g.15532 C>A, g.16907 T>C & 1.0000 & 0.9886 & $10 / 10$ \\
\hline & g.12870 T>C, g.13126 T>C, g.15532 C>A, g.16907 T>C, g.17924 G>A & 1.0000 & 0.9744 & $10 / 10$ \\
\hline
\end{tabular}

SNP, single nucleotide polymorphism; MS, marbling score; MDR, multifactor dimensionality reduction.

${ }^{1)}$ Training-balanced accuracy. ${ }^{2)}$ Test-balanced accuracy. ${ }^{3)}$ Cross-validation consistency. 
Table 4. The best SNP in five-factor interactions of the CWT base in the MDR method for Korean native cattle

\begin{tabular}{|c|c|c|c|c|}
\hline Adjustment & Model & Training acc. ${ }^{1)}$ & Test acc. ${ }^{2)}$ & $\mathrm{CVC}^{3)}$ \\
\hline \multirow[t]{5}{*}{ Before } & g. $15532 C>A$ & 0.5212 & 0.4442 & $5 / 10$ \\
\hline & g. $12870 \mathrm{~T}>\mathrm{C}, \mathrm{g} .15532 \mathrm{C}>\mathrm{A}$ & 0.5441 & 0.4479 & $8 / 10$ \\
\hline & g. $13126 \mathrm{~T}>\mathrm{C}$, g. $15532 \mathrm{C}>\mathrm{A}, \mathrm{g} .17924 \mathrm{G}>\mathrm{A}$ & 0.5664 & 0.4829 & $7 / 10$ \\
\hline & g.13126 T>C, g.15532 C > A, g.16907 T>C, g.17924 G > A & 0.5781 & 0.4847 & $6 / 10$ \\
\hline & g.12870 T>C, g.13126 T>C, g.15532 C>A, g.16907 T>C, g.17924 G>A & 0.5865 & 0.4770 & $10 / 10$ \\
\hline \multirow[t]{5}{*}{ After } & g. $12870 \mathrm{~T}>\mathrm{C}$ & 0.8440 & 0.8440 & $10 / 10$ \\
\hline & g.12870 T>C, g. $17924 \mathrm{G}>\mathrm{A}$ & 0.9177 & 0.9054 & $8 / 10$ \\
\hline & g.12870 T>C, g.16907 T>C, g.17924 G>A & 0.9484 & 0.9351 & $9 / 10$ \\
\hline & g.12870 T>C, g.13126 T>C, g.15532 C >A, g.17924 G>A & 0.9651 & 0.9612 & $10 / 10$ \\
\hline & g.12870 T>C, g.13126 T>C, g.15532 C>A, g.16907 T>C, g.17924 G>A & 1.0000 & 0.9922 & $10 / 10$ \\
\hline
\end{tabular}

SNP, single nucleotide polymorphism; CWT, carcass weight; MDR, multifactor dimensionality reduction.

${ }^{1)}$ Training-balanced accuracy. ${ }^{2)}$ Test-balanced accuracy. ${ }^{3)}$ Cross-validation consistency.

factor interaction between g.12870 T>C and g.17924 G>A showed the highest accuracy at $90.54 \%$ (Table 4 ).

Third, gene-gene interactions of C18:1, MUFAs, MS, and CWT were investigated using a detailed MDR interaction model, and the two-factor interaction was selected as the best for each economic trait (Figure 1). Each genotype was divided into a high- or low-risk group in the selected two-factor interaction. If the casecontrol ratio for each genotype was higher than the threshold, then the genotype was identified as a high-risk group. In addition, mean scores between high- and low-risk groups were compared (Table 5). The thresholds of C18:1 and MUFAs were 1.2599 and 1.2208, respectively. Each case-control ratio for TTCC, TTCA, TTAA, TCCA, TCAA, and CCAA for the interaction between g.13126 T>C and g.15532 C >A was above the respective threshold; these six genotypes were therefore classified as the high-risk group and the others as the low-risk group. According to the threshold (1.9148) of MS, TTAA, TCCA, TCAA, CCCC, CCCA, and CCAA, the interaction between g.12870 $\mathrm{T}>\mathrm{C}$ and g.15532 C $>\mathrm{A}$ was classified as the high-risk group. In the case of CWT, the threshold was 0.3359 , and therefore the TCGA of the g.12870 $\mathrm{T}>\mathrm{C}$ and g.17924 G>A interaction was identified as the high-risk group.

Finally, high- and low-risk genotypes were compared based on their mean scores for each trait (Table 5). Table 5 compares individual SNP and interaction effects. The t-test was significant $(\mathrm{p}<0.001)$, and the effect size of the genotype of the SNP interaction was larger than that of the individual SNP.

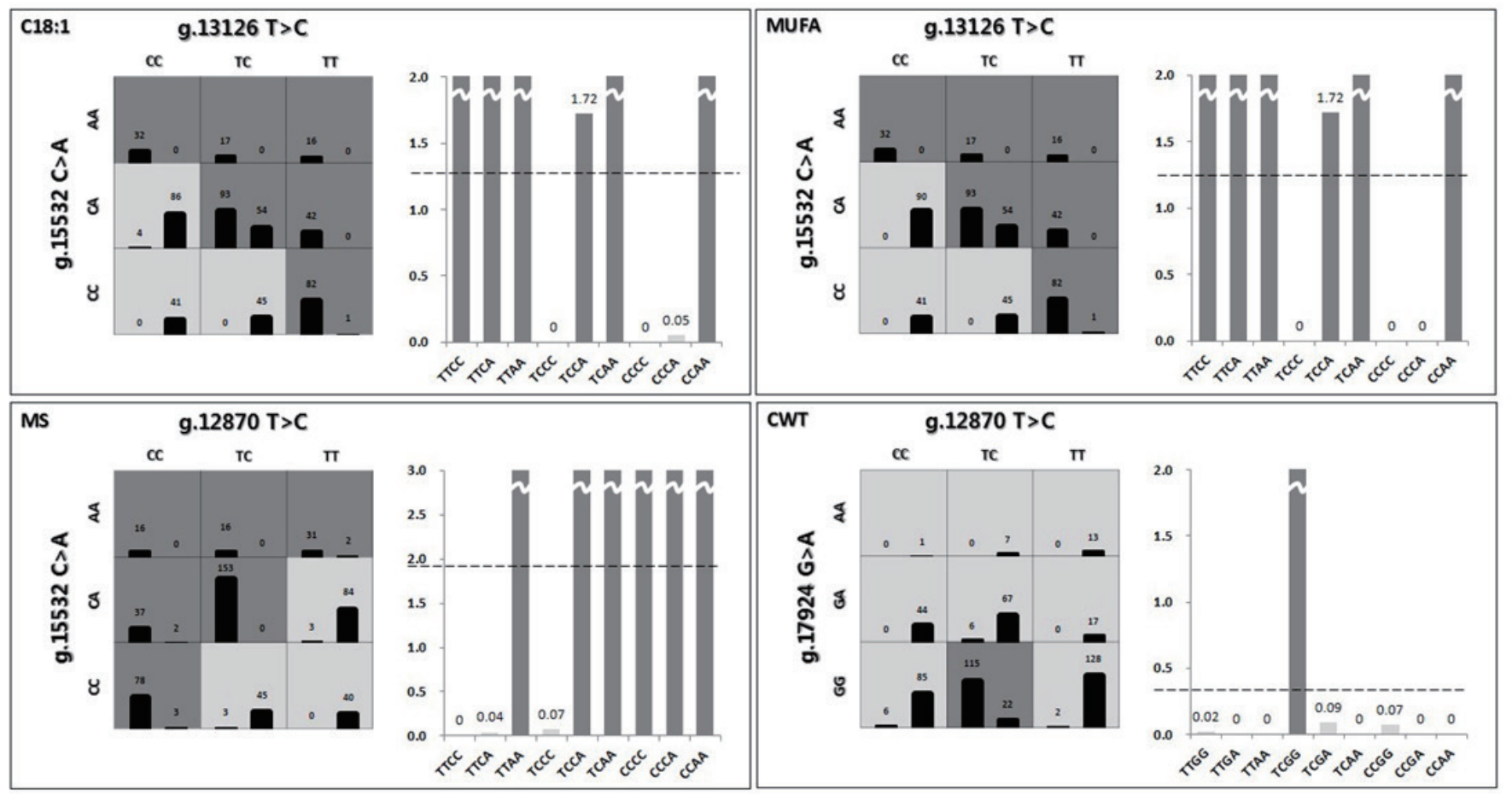

Figure 1. The best two-factor interaction for four traits based on the multifactor dimensionality reduction (MDR) method for Korean native cattle. The high-risk group is indicated by dark grey, and the low-risk group, by grey. The left bar of a cell represents case frequency, and the right bar, control frequency. 
Table 5. Comparison of mean scores between high- and low-risk groups of Korean native cattle

\begin{tabular}{|c|c|c|c|c|c|c|c|}
\hline Traits & SNP & Genotype & $\mathrm{N}$ & Mean & SD & $\begin{array}{c}\text { t-test } \\
\mathrm{p} \text {-value }\end{array}$ & Cohen's d \\
\hline \multirow[t]{6}{*}{ C18:1 } & g.13126 T>C & $\mathrm{TT}$ & 141 & 44.832 & 0.929 & 6.982 & 1.681 \\
\hline & & $\mathrm{TC}, \mathrm{CC}$ & 372 & 43.155 & 1.061 & $(<0.001)$ & \\
\hline & g.15532C >A & AA & 65 & 45.123 & 0.966 & 4.840 & 1.620 \\
\hline & & $C A, A A$ & 448 & 43.397 & 1.156 & $(<0.001)$ & \\
\hline & g. $13126 \mathrm{~T}>\mathrm{C}, \mathrm{g} .15532 \mathrm{C}>\mathrm{A}$ & TTCC, TTCA, TTAA, TCCA, TCAA , CCAA & 337 & 44.238 & 0.896 & 20.866 & 1.905 \\
\hline & & Others & 176 & 42.425 & 1.005 & $(<0.001)$ & \\
\hline \multirow[t]{6}{*}{ MUFA } & g.13126 T>C & $\mathrm{TT}$ & 141 & 53.799 & 0.902 & 6.528 & 1.783 \\
\hline & & $\mathrm{TC}, \mathrm{CC}$ & 372 & 52.023 & 1.083 & $(<0.001)$ & \\
\hline & g.15532C>A & $\mathrm{AA}$ & 65 & 54.107 & 0.985 & 4.747 & 1.684 \\
\hline & & $\mathrm{CA}, \mathrm{CC}$ & 448 & 52.279 & 1.177 & $(<0.001)$ & \\
\hline & g. $13126 \mathrm{~T}>\mathrm{C}, \mathrm{g} .15532 \mathrm{C}>\mathrm{A}$ & TTCC, TTCA, TTAA, TCCA, TCAA, CCAA & 337 & 53.162 & 0.911 & 21.600 & 1.977 \\
\hline & & Others & 176 & 51.265 & 1.005 & $(<0.001)$ & \\
\hline \multirow[t]{6}{*}{ MS } & g.12870 T>C & TT, TC & 353 & 5.749 & 0.397 & 9.829 & 0.980 \\
\hline & & $\mathrm{CC}$ & 160 & 5.300 & 0.511 & $(<0.001)$ & \\
\hline & g. 15532 C >A & $C A, A A$ & 344 & 5.765 & 0.359 & 10.287 & 1.029 \\
\hline & & $\mathrm{CC}$ & 169 & 5.291 & 0.544 & $(<0.001)$ & \\
\hline & g. $12870 \mathrm{~T}>\mathrm{C}, \mathrm{g} .15532 \mathrm{C}>\mathrm{A}$ & TTAA, TCCA, TCAA, CCCC, CCCA, CCAA, & 338 & 5.846 & 0.300 & 18.843 & 1.852 \\
\hline & & Others & 175 & 5.151 & 0.437 & $(<0.001)$ & \\
\hline \multirow[t]{6}{*}{ CWT } & g.12870 T>C & TC & 217 & 429.997 & 5.802 & 7.528 & 0.688 \\
\hline & & $\mathrm{TT}, \mathrm{CC}$ & 296 & 425.227 & 7.902 & $(<0.001)$ & \\
\hline & g.17924 G>A & GG & 358 & 429.327 & 6.656 & 10.590 & 1.007 \\
\hline & & $\mathrm{GA}, \mathrm{AA}$ & 155 & 422.436 & 7.021 & $(<0.001)$ & \\
\hline & g. $12870 \mathrm{~T}>\mathrm{C}$, g. $17924 \mathrm{G}>\mathrm{A}$ & TCGA & 137 & 432.251 & 3.787 & 13.394 & 1.132 \\
\hline & & Others & 376 & 425.421 & 7.643 & $(<0.001)$ & \\
\hline
\end{tabular}

SNP, single nucleotide polymorphism; SD, standard deviation; MUFA, monounsaturated fatty acid; MS, marbling score; CWT, carcass weight.

\section{DISCUSSION}

To determine the interaction effect of Hanwoo, we used five SNPs which are known as the factors affecting the flavor and quality of beef [18]. Phenotypes are influenced by environmental and genetic factors. To enhance the accuracy of the genetic effect analysis, the study proposes a new statistical model that excludes environmental factors based on an ANCOVA model. The statistical model has qualitative independent variable which has more than two classes (17 farms). Then, we tried additional indicator variables in the model [14]. The results verify a significant increase in analysis accuracy.

Data from 513 Hanwoo steer from 17 farms in Gyengsangbukdo were employed to construct an ANCOVA model of how economic traits are influenced by five SNPs and environmental factors. Here economic traits were C18:1, MUFAs, MS, and CWT and five SNPs (g.12870 T>C, g.13126 T>C, g.15532 C>A, g.16907 $\mathrm{T}>\mathrm{C}$, and g.17924 G>A), which we previously found to influence the flavor and quality of beef [7]. Some of these SNPs might change their functions by producing missense codons. The g.13126 $\mathrm{T}<\mathrm{C}$ changes an amino acid from tyrosine to histidine. The g.15532 $\mathrm{C}<\mathrm{A}$ or g.17924 $\mathrm{G}<\mathrm{A}$ changes an amino acid from leucine to isoleucine or from alanine to threonine. Although the other SNPs are synonymous, we could not rule out the possibility that they might change splicing regulatory sequences, or that such genetic associations might be spuriously produced with their linked loci within each block [18].

Then, we constructed an adjusted statistical model using an ANCOVA model and calculated adj $(\mathrm{Y})$ values. The MDR method was used for individual SNPs and five-factor interactions for each economic trait (C18:1, MUFAs, MS, and CWT). According to the results, the accuracy of SNP interactions was much higher than that of individual SNPs. The most significant two-factor interaction was g.13126 T>C $C^{\star}$ g.15532 C $>\mathrm{A}$ for C18:1 and MUFAs. In addition, the g.12870 $\mathrm{T}>\mathrm{C}^{\star} \mathrm{g} .15532 \mathrm{C}>\mathrm{A}$ interaction showed the highest accuracy in MS, and the g.12870 T>C ${ }^{\star}$ g.17924 G>A interaction showed the highest accuracy in CWT. The three SNPs in exons 34 (g.15532 C<A), 37 (g.16907 T<C), and 39 (g.17924 $\mathrm{G}<\mathrm{A}$ ) were all associated with myristic acid in a crossbred of Limousin and Jersey $(\mathrm{p}<0.05)$ but not with C18:1 [8]. The g.17924 was associated also with oleic acid in American Angus cattle $(\mathrm{p}<0.05)[10]$ and Korean cattle $(\mathrm{p}<0.05)$ [19]. This implies that these two-factor interactions had the greatest impact on economic traits influencing the flavor and quality of beef. The selected interaction for CWT changed before adjustment because CWT was heavily influenced by environmental factors. Figure 1 shows the contingency tables of the best two-factor interactions for each economic trait. The chi-square test of interactions was conducted to validate the results, and all tests were significant. In the figure, three factors and their possible multifactor classes or cells are 
represented in a three-dimensional space [20]. The left bar of the cell refers to case frequency, and the right bar, to control frequency. Dark grey cells and grey cells indicate "high-risk" and "low-risk" groups, respectively, depending on whether the casecontrol ratio was above a certain threshold. The threshold was determined based on the case-control ratio for the whole data. Because the data were passed through the k-means clustering method for each trait, they had three different case-control ratios. Therefore, cells were classified based on the threshold for each trait. The high- and low-risk groups are shown in the graphs. The threshold was 1.2599 in C18:1. Nine genotypes of the two-factor interaction were divided into two groups by comparing their case-control ratios and thresholds. TTCC, TTCA, TTAA, TCCA, TCAA, and CCAA were labeled as the high-risk group and the others as the low-risk group. In MUFAs, MS, and CWT, the thresholds were $1.2208,1.9148$, and 0.3359 , respectively. Similarly, each genotype was determined by comparing its ratio and threshold. TTCC, TTCA, TTAA, TCCA, TCAA, and CCAA for MUFAs; TTAA, TCCA, TCAA, CCCC, CCCA, and CCAA for MS; and TCGA for CWT were classified as the high-risk group. There was a significant difference between the high- and lowrisk groups (Table 5), indicating that high-risk genotypes were superior. In particular, two-factor interactions showed clear differences between the high- and low-risk groups. According to the results, the gene-gene interaction effect was superior to the individual SNP effect. As shown in Table 5, individual and interaction effects of SNPs were examined and compared for C18:1, MUFAs, MS, and CWT. The superior genotype groups, which were all and significant, were 44.238 (standard deviation $[\mathrm{SD}]=0.896, \mathrm{p}<0.001$, Cohen's $\mathrm{d}=1.905)$ for C18:1, 53.162 (SD $=0.911, \mathrm{p}<0.001$, Cohen's $\mathrm{d}=1.977)$ for MUFAs, $5.846(\mathrm{SD}=0.3$, $\mathrm{p}<0.001$, Cohen's $\mathrm{d}=1.852)$ for MS, and $432.251(\mathrm{SD}=3.787$, $p<0.001$, Cohen's $d=1.132$ ) for CWT (Table 5). The interaction effect of SNPs was greater than the individual effect for C18:1, MUFAs, MS, and CWT.

The results verify that the proposed MDR nonparametric statistical method for detecting gene interactions is suitable for small samples and can be used to perform an exhaustive search of all $\mathrm{n}$-locus models by collapsing multi-locus genotypes into highand low-risk groups [20]. The MDR method may be used for the genetic assessment of quantitative traits after further development. Finally, these genotypes of individual SNPs and their combinations may be useful genetic markers for improving beef quality, which is one of the most important goals of the Korean beef cattle industry [21].

\section{CONFLICT OF INTEREST}

We certify that there is no conflict of interest with any financial organization regarding the material discussed in the manuscript.

\section{ACKNOWLEDGMENTS}

Jea-Young Lee's work was supported by a 2015 Yeungnam University Research Grant.

\section{REFERENCES}

1.Westerling DB, Hedrick HB. Fatty acid composition of bovine lipids as influenced by diet, sex and anatomical location and relationship to sensory characteristics. Anim Sci J 1979;48:1343-8.

2.Melton SL, Amiri M, Davis GW, Backus WR. Flavor and chemical characteristics of ground beef from grass-, forage-grain and grainfinished steers. Anim Sci J 1982;55:77-87.

3.Matsumoto N, Kakihara F, Kimura S, et al. Single nucleotide polymorphism genotyping of CYP2C19 using a new automated system. Anal Biochem 2007;370:121-3.

4.Sturdivant CA, Lunt DK, Smith GC, Smith SB. Fatty acid composition of subcutaneous and intramuscular adipose tissues and $M$. longissimus dorsi of Wagyu cattle. Meat Sci 1991;32:449-58.

5.Mandell IB, Buchanan-Smith JG, Campbell CP. Effects of forage vs. grain feeding on carcass characteristics, fatty acid composition, and beef quality in Limousin-cross steers when time on feed is controlled. Anim Sci J 1998;76:2619-30.

6.Roy R, Taourit R, Zaragoza P, Eggen A, Rodellar C. Genomic structure and alternative transcript of bovine fatty acid synthase gene (FASN): comparative analysis of the FASN gene between monogastric and ruminant species. Cytogenet Genome Res 2005;111:65-73.

7.Oh D, Lee Y, La B, et al. Fatty acid composition of beef is associated with exonic nucleotide variants of the gene encoding FASN. Mol Biol Rep 2012;39:4083-90.

8.Morris CA, Cullen NG, Glass BC, et al. Fatty acid synthase effects on bovine adipose fat and milkfat. Mamm Genome 2007;18:64-74.

9.Abe T, Saburi J, Hasebe $\mathrm{H}$, et al. Novel mutations of the FASN gene and their effect on fatty acid composition in Japanese black beef. Biochem Genet 2009;47:397-411.

10. Zhang S, Knight TJ, Reecy JM, Beitz DC. DNA polymorphisms in bovine fatty acid synthase are associated with beef fatty acid composition. Anim Genet 2008;39:62-70.

11. Inoue K, Shoji N, Kobayashi M. Genetic relations among fat melting point, fatty acid composition and carcass traits in Japanese Black cattle. Nihon Chikusan Gakkaiho 2008;79:1-8.

12. Folch J, Lees M, Sloane Stanley GH. A simple method for the isolation and purification of 12 total lipids from animal tissue. J Biol Chem 1957;226:497-509.

13. O'Keefe PW, Wellington GH, Mattick LR, Stouffer JR. Composition of bovine muscle lipids at various carcass locations. J Food Sci 1968;33: 188-92.

14. Neter J, Wasserman W, Kutner MH. Applied linear statistical models. 3th ed. Boston, MA: Irwin; 1990.

15. Hahn LW, Ritchie MD, Moore JH. Multifactor dimensionality reduction software for detecting gene-gene and gene-environment interactions. Bioinformatics 2003;19:376-82. 
16. McGraw KO, Wong SP. A common language effect size statistic. Psychological. Bulletin 1992;111:361-5.

17. Cohen J. Statistical power analysis for the behavioral sciences.2th ed. Hillsdale, NJ: Lawrence Erlbaum Associates; 1998.

18. Lee J, Jin M, Lee Y, et al. Gene-gene interactions of fatty acid synthase (FASN) using multifactor-dimensionality reduction method in Korean cattle. Mol Biol Rep 2014;41:2021-7.

19. Bhuiyan MSA, Yu SL, Jeon JT, et al. DNA polymorphisms in SREBF1 and FASN genes affect fatty acid composition in Korean cattle (Hanwoo). Asian-Australas J Anim Sci 2009;22:765-73.

20. Ritchie MD, Hahn LW, Roodi N, et al. Multifactor-dimensionality reduction reveals high-order interactions among estrogen- metabolism genes in sporadic breast cancer. Am J Hum Genet 2001;69:138-47.

21. Kim J, Kim D, Lee J, Lee C. Genetic relationship between carcass traits and carcass price of Korean cattle. Asian-Australas J Anim Sci 2010;23: 848-54. 